\title{
Numerical Solution of Second-Order Linear Difference Equations
}

\author{
F. W. J. Olver \\ Institute for Basic Standards, National Bureau of Standards, Washington, D.C. 20234
}

(May 4, 1967)

\begin{abstract}
A new algorithm is given for computing the solution of any second-order linear difference equation which is applicable when simple recurrence procedures cannot be used because of instability. Compared with the well-known Miller algorithm the new method has the advantages of (i) automatically determining the correct number of recurrence steps, (ii) applying to inhomogeneous difference equations, (iii) enabling more powerful error analyses to be constructed.

The method is illustrated by numerical computations, including error analyses, of Anger-Weber, Struve, and Bessel functions, and the solution of a differential equation in Chebyshev series.

Key Words: Chebyshev series, difference equations, error analysis, Miller algorithm, recurrence methods, special functions.
\end{abstract}

\section{Introduction}

A powerful computational algorithm for evaluating the most rapidly decreasing solution of a second-order homogeneous linear difference equation was published in 1952 by J. C. P. Miller ([1], ${ }^{1}$ page xvii) in connection with the tabulation of modified Bessel functions. Since then, various writers have applied the algorithm to other special functions, and similar computational processes have been used by Clenshaw [2] for the numerical solution of ordinary differential equations in series of Chebyshev polynomials. Error analyses of the algorithm have been supplied by the present writer [3] and Oliver [12] and quite recently Gautschi [4] has examined the relation of the algorithm to classical results in the theory of continued fractions.

The present investigation stems from the observation that Miller's algorithm can be regarded as a procedure for solving a tridiagonal set of simultaneous linear algebraic equations. Adopting this more general standpoint, we shall show how to recast the algorithm into a new form which enables the correct number of recurrence steps to be determined automatically without appeal to an asymptotic or other analytical formula. In this respect it resembles an algorithm proposed recently by Shintani [5].

The new formulation has the further advantages of (i) being applicable to inhomogeneous difference equations, (ii) lending itself readily to powerful error analyses. There seems to be no alternative method of comparable power available at present for computing solutions of inhomogeneous equations in the case when forward recurrence and backward recurrence are both unstable.

\section{Statement of the Problem}

Let the given difference equation be denoted by

$$
a_{r} y_{r-1}-b_{r} y_{r}+c_{r} y_{r+1}=d_{r},
$$

'Figures in brackets indicate the literature references at the end of this paper. 
where $a_{r}, b_{r}, c_{r}$, and $d_{r}$ are given functions of the nonnegative integer variable $r$. We assume that the general solution of (2.01) has the form

$$
y_{r}=A f_{r}+B g_{r}+h_{r},
$$

in which $A$ and $B$ are arbitrary constants, and the complementary functions $f_{r}, g_{r}$, and the particular solution $h_{r}$ have the properties $f_{0} \neq 0, g_{r} \neq 0$ for all sufficiently large $r$, and

$$
f_{r} / g_{r} \rightarrow 0, \quad h_{r} / g_{r} \rightarrow 0, \quad(r \rightarrow \infty) .
$$

(It may be noted that we do not require either $f_{r}$ or $h_{r}$ to tend to zero as $r \rightarrow \infty$.)

The first problem we investigate is the computation of the solution of (2.01) which has the property

$$
y_{r} / g_{r} \rightarrow 0 \quad(r \rightarrow \infty),
$$

and satisfies the normalizing condition

$$
y_{0}=k
$$

for an arbitrarily assigned value of the constant $k$. Later (secs. 9-11) we allow for a more general form of normalizing condition and also drop the restriction $f_{0} \neq 0$.

The given conditions ensure that $y_{r}$ exists and is unique. For, from (2.03) and (2.04) the $B$ of (2.02) is seen to be zero, and from (2.05) we derive $A=\left(k-h_{0}\right) / f_{0}$. Therefore

$$
y_{r}=\frac{k-h_{0}}{f_{0}} f_{r}+h_{r} .
$$

It is well known that direct use of (2.01) as a recurrence relation for generating $y_{2}, y_{3}, \ldots$. from given values of $y_{0}$ and $y_{1}$ (if available) is an unstable procedure. Essentially, each computational rounding error introduces into the numerical solution a small multiple of $f_{r}$ and a small multiple of $g_{r}$, and in consequence of (2.04) the latter ultimately grows faster than the wanted solution.

It may also happen ${ }^{2}$ in the inhomogeneous case that $f_{r}$ grows more rapidly than $y_{r}$ in the direction of decreasing $r$. In this event recurrence by use of (2.01) is unstable in this direction too.

\section{Approach}

Analogous work in the numerical solution of linear differential equations ${ }^{3}$ suggests that a stable way of solving the present problem is to treat it directly as a boundary-value problem rather than use initial-value techniques. We are already given the value of $y_{0}$. Suppose that for some large integer $N$, the value of $y_{N}$ can be obtained from an asymptotic formula or by other means. Then eqs (2.01) with $r=1,2, \ldots, N-1$ comprise a set of simultaneous linear algebraic equations for the unknowns $y_{1}, y_{2}, \ldots ., y_{N-1}$, which are solvable by standard matrix computational processes.

This possibility has already been noted by Gautschi ([4], Introduction) following a suggestion by M. E. Rose, but Gautschi did not pursue the idea because of the difficulty of obtaining the value of $y_{N}$, in general. Following Miller's approach in the homogeneous case [1], we solve the algebraic equations with the value of $y_{N}$ arbitrarily set equal to zero. It transpires that for large $N$ the great majority of the $y_{r}$ produced in this way are generally excellent approximations to the true values; only in the neighborhood of $r=N$ can substantial errors occur.

We first establish the convergence of the process.

\footnotetext{
${ }^{2}$ See Example 1 of section 6

${ }^{3}$ See, for example, [6].
} 
THEOREM 1. With the conditions of section 2, suppose that for all sufficiently large $\mathrm{N}$ the system of equations

$$
a_{r} y_{r-1}^{(N)}-b_{r} y_{r}^{(N)}+c_{r} y_{r+1}^{(N)}=d_{r}, \quad(r=1,2, \ldots, N-1)
$$

and

$$
\mathrm{y}_{0}^{(\mathrm{N})}=\mathrm{k}, \quad \mathrm{y}_{\mathrm{N}}^{(\mathrm{N})}=0,
$$

has a solution $\mathrm{y}_{0}^{(\mathrm{N})}, \mathrm{y}_{1}^{(\mathrm{N})}, \ldots, \mathrm{y}_{\mathrm{N}}^{(\mathrm{N})}$. Then if $\mathrm{r}$ is fixed and $\mathrm{N} \rightarrow \infty, \mathrm{y}_{\mathrm{r}}^{(\mathrm{N})} \rightarrow \mathrm{y}_{\mathrm{r}}$.

To establish this result, we observe that since $y_{r}$ is a particular solution of (2.01), we can express

$$
y_{r}^{(N)}=A_{N} f_{r}+B_{N} g_{r}+y_{r}
$$

where $A_{N}$ and $B_{N}$ are independent of $r$. Setting $r=0, N$ in turn and using (2.05) and (3.02), we derive

$$
A_{N}=\frac{g_{0} y_{N}}{f_{0} g_{N}-g_{0} f_{N}}, \quad B_{N}=-\frac{f_{0} y_{N}}{f_{0} g_{N}-g_{0} f_{N}}
$$

the denominator $f_{0} g_{N}-g_{0} f_{N}$ being nonvanishing for all sufficiently large $N$ in consequence of the assumed conditions. In fact, from (2.03) we have

$$
f_{0} g_{N}-g_{0} f_{N} \sim f_{0} g_{N} \quad(N \rightarrow \infty) .
$$

Hence from (2.04) it follows that $A_{N} \rightarrow 0$ and $B_{N} \rightarrow 0$. This completes the proof.

Thus for any given value of $r$, or for any finite range of values, $y_{r}$ can be calculated to prescribed accuracy by solving the system of equations (3.01) and (3.02) with a sufficiently high value of $N$. Naturally, we now enquire what exactly constitutes a "sufficiently high" value? Or, to put the question another way, given $N$, to what accuracy does $y_{r}^{(N)}$ approximate $y_{r}$ for $r<N$ ?

A simple practical way of providing an answer is to solve eqs (3.01) and (3.02) for increasing values of $N$ until the results are in satisfactory numerical agreement. This procedure has two drawbacks. First, it is wasteful of computing time if the originally guessed values of $N$ are either too low or much too high. Second, there is no absolute guarantee that values of $y_{r}$ computed with two (or more) different values of $N$ must be correct when they agree.

The optimum value of $N$, that is, the minimum value necessary to achieve specified accuracy in $y_{r}$ for a given range of values of $r$, can be determined automatically when a suitable method is used to solve the algebraic equations. Accordingly, we consider this process next.

\section{Solution of the Algebraic Equations}

We shall solve the tridiagonal system of equations

$$
\begin{aligned}
& -b_{1} y_{1}^{(N)}+c_{1} y_{2}^{(N)} \\
& a_{2} y_{1}^{(N)}-b_{2} y_{2}^{(N)}+c_{2} y_{3}^{(N)} \\
& =d_{1}-a_{1} k \text {, } \\
& a_{3} y_{2}^{(N)}-b_{3} y_{3}^{(N)}+c_{3} y_{4}^{(N)} \\
& =d_{2} \text {, } \\
& =d_{3} \text {, } \\
& a_{N-2} y_{N-3}^{(N)}-b_{N-2} y_{N-2}^{(N)}+c_{N-2} y_{N-1}^{(N)}=d_{N-2}, \\
& a_{N-1} y_{N-2}^{(N)}-b_{N-1} y_{N-1}^{(N)}=d_{N-1}
\end{aligned}
$$


by simple elimination followed by back-substitution. To begin with we suppose that none of the $c_{r}$ vanish.

Let the first of (4.01) be rewritten in the form

$$
p_{2} y_{1}^{(N)}-p_{1} y_{2}^{(N)}=e_{1}
$$

where

$$
p_{1}=1, \quad p_{2}=\frac{b_{1}}{c_{1}}, \quad e_{1}=\frac{a_{1} k-d_{1}}{c_{1}} .
$$

The result of eliminating $y_{1}^{(N)}$ from (4.02) and the second of (4.01) can be expressed as

$$
p_{3} y_{2}^{(N)}-p_{2} y_{3}^{(N)}=e_{2}
$$

where

$$
p_{3}=\frac{b_{2} p_{2}-a_{2} p_{1}}{c_{2}}, \quad e_{2}=\frac{a_{2} e_{1}-d_{2} p_{2}}{c_{2}}
$$

Continuing the elimination, we obtain

$$
p_{r+1} y_{r}^{(N)}-p_{r} y_{r+1}^{(N)}=e_{r} \quad(r=1,2, \ldots, N-1)
$$

where

$$
p_{r+1}=\frac{b_{r} p_{r}-a_{r} p_{r-1}}{c_{r}}, \quad e_{r}=\frac{a_{r} e_{r-1}-d_{r} p_{r}}{c_{r}}
$$

Thus $p_{r}$ is the solution of the homogeneous form of the difference eq (2.01), with the initial conditions $p_{0}=0$ and $p_{1}=1$. We also observe that the second of (4.05) holds for $r=1$ if we define

$$
e_{0}=k \text {. }
$$

The final equation of the form (4.04) is used to begin the back-substitution. On substituting the second of (3.02), we derive

$$
y_{N-1}^{(N)}=e_{N-1} / p_{N}
$$

thence $y_{N-2}^{(N)}, y_{N-3}^{(N)}, \ldots ., y_{1}^{(N)}$ may be computed by use of (4.04) with descending values of $r$. The process fails if, and only if, one of the numbers $p_{2}, p_{3}, \ldots ., p_{N}$ vanishes. In this event the set of eqs (3.01) and (3.02) has either no solution or an infinity of solutions, and the algorithm breaks down.

When one or more of the coefficients $c_{r}$ vanishes the set of eqs (4.01) becomes uncoupled. A simple modification takes care of the situation. Suppose, for example, that $c_{s}=0$ but all other $c_{r}$ are nonzero. Then the first $s$ equations of (4.01) determine $y_{1}^{(N)}, y_{2}^{(N)}, \ldots ., y_{s}^{(N)}$ completely: they can be solved by application of the recurrence relations (4.05) for $r=1,2, \ldots, s-1$ and use of the back-substitution relation (4.04), beginning with

$$
y_{s}^{(N)}=\frac{a_{s} e_{s-1}-d_{s} p_{s}}{b_{s} p_{s}-a_{s} p_{s-1}}
$$


The remaining $N-s-1$ equations are solvable for $y_{s+1}^{(N)}, y_{s+2}^{(N)}, \ldots ., y_{N-1}^{(N)}$ by the method already described: eq (4.07) takes the place of the first of (3.02).

To ease the presentation we shall suppose in the remainder of the paper that none of the $c_{r}$ vanish.

Applying ourselves to the problem of determining the optimum value of $N$, we observe that the effect of replacing $N$ by $N+1$ is to prolong the elimination process by one step, beginning the back-substitution with $y_{N+1}^{(N+1)}=0$ instead of $y_{N}^{(N)}=0$. Thus we have

$$
p_{r+1} y_{r}^{(N+1)}-p_{r} y_{r+1}^{(N+1)}=e_{r} \quad(r=1,2, \ldots, N) .
$$

Subtraction of (4.04) from (4.08) gives

$$
y_{r}^{(N+1)}-y_{r}^{(N)}=\frac{p_{r}}{p_{r+1}}\left(y_{r+1}^{(N+1)}-y_{r+1}^{(N)}\right) \quad(r \leqslant N-1),
$$

and repeated application of this result leads to

$$
y_{r}^{(N+1)}-y_{r}^{(N)}=\frac{p_{r}}{p_{r+1}} \frac{p_{r+1}}{p_{r+2}} \cdots \frac{p_{N-1}}{p_{N}}\left(y_{N}^{(N+1)}-y_{N}^{(N)}\right),
$$

that is,

$$
y_{r}^{(N+1)}-y_{r}^{(N)}=\frac{p_{r} e_{N}}{p_{N} p_{N+1}} \quad(r=1,2, \ldots, N) .
$$

By use of this formula we can predict the effect of changing $N$ into $N+1$ before any back-substitution is carried out.

Suppose, for example, that we wish to compute $y_{L}$ to $D$ decimal places for given values of the integers $L$ and $D$. Then the recurrence relations (4.05) are applied from $r=1$ past $r=L$ until a value of $r$ is reached for which

$$
\left|\frac{p_{L} e_{r}}{p_{r} p_{r+1}}\right|<\frac{1}{2} \times 10^{-D}
$$

If this value of $r$ is taken as $N$, then we can be sure that the approximation $y_{L}^{(N)}$ yielded by the back-substitution agrees to $D$ decimal places with the value $y_{L}^{(N+1)}$ that would be obtained from the next higher approximation. (Whether this value of $N$ is adequate is considered in the next section.)

If, as is more usual, accurate values of $y_{r}$ are required for a whole range of values of $r$, then the criterion (4.11) is used with $\left|p_{L}\right|$ denoting the greatest value of $\left|p_{r}\right|$ in the given range. We might, for example, desire the computation to $D$ decimal places of all values of $y_{r}$ that exceed $1 / 2 \times 10^{-D}$ in absolute value - it being assumed, of course, in this case that $y_{r} \rightarrow 0$ as $r \rightarrow \infty$. Then $N$ is determined by the condition that

$$
\left|e_{N}\right| p_{N+1} \mid<\frac{1}{2} \times 10^{-D},
$$

provided that $\left|p_{N}\right| \geqslant\left|p_{r}\right|$ when $r<N$.

\section{Expansions for the Solution and the Truncation Error}

The method suggested in the last section for determining $N$ is based on the criterion that the values of $y_{r}^{(N)}$ and $y_{r}^{(N+1)}$ must agree to within the prescribed tolerance for $y_{r}$. This does not guarantee, however, that their common value is $y_{r}$. To resolve this doubt we consider higher approximations. 
Replacing $N$ by $N+1$ in (4.10), and adding the result to (4.10) itself, we obtain

$$
y_{r}^{(N+2)}-y_{r}^{(N)}=p_{r}\left(\frac{e_{N}}{p_{N} p_{N+1}}+\frac{e_{N+1}}{p_{N+1} p_{N+2}}\right) .
$$

Continuation of this process yields

$$
y_{r}^{(N+s)}-y_{r}^{(N)}=p_{r}\left(\frac{e_{N}}{p_{N} p_{N+1}}+\frac{e_{N+1}}{p_{N+1} p_{N+2}}+\ldots+\frac{e_{N \vdash s-1}}{p_{N+s-1} p_{N+s}}\right),
$$

where $s$ is an arbitrary positive integer. Letting $s \rightarrow \infty$ and using Theorem 1, we derive the following expression for the truncation error

$$
\epsilon_{r}^{(N)} \equiv y_{r}-y_{r}^{(N)}=E_{N} p_{r}
$$

where $E_{N}$ is the sum of the (necessarily convergent) series

$$
E_{N}=\sum_{s=N}^{\infty} \frac{e_{s}}{p_{s} p_{s+1}}
$$

Thus the precise criterion for determining $N$ is that $\left|E_{N} p_{r}\right|$ must not exceed the specified tolerance in $y_{r}$ for each wanted value of $r$.

Once the value of $N$ has been decided, the actual value of the truncation error can be found by continuing the computation of $p_{r}$ and $e_{r-1}$ beyond $r=N$ and using (5.01) and (5.02). Later [7], we shall show how to use these expansions to determine strict bounds for $\epsilon_{r}^{(N)}$ directly from the properties of the coefficients $a_{r}, b_{r}, c_{r}$, and $d_{r}$.

As a special case of (5.01) we have the expansion

$$
y_{r}=p_{r} \sum_{s=r}^{\infty} \frac{e_{s}}{p_{s} p_{s+1}} .
$$

Subtraction of (5.01) from (5.03) yields

$$
y_{r}^{(N)}=p_{r} \sum_{s=r}^{N-1} \frac{e_{s}}{p_{s} p_{s+1}} \quad(r<N),
$$

a result which is obtainable more directly by repeated use of the back-substitution relation (4.04). Thus the whole of our computing scheme is equivalent to approximating the convergent infinite series (5.03) by the partial sum (5.04).

\section{Examples}

EXAmple 1. Anger-Weber functions.

For integer values of $r$ the function $\mathbf{E}_{r}(x)$ satisfies eq (2.01) with

$$
a_{r}=c_{r}=1, \quad b_{r}=\frac{2 r}{x}, \quad d_{r}=-\frac{2\left\{1-(-1)^{r}\right\}}{\pi x} .
$$

We restrict ourselves here to positive values of the argument $x$. The principal properties of $\mathbf{E}_{r}(x)$ are established in [8], chapter 10. In particular, we have

$$
\mathbf{E}_{2 r}(x)=\frac{2 x}{\left(4 r^{2}-1\right) \pi} \sum_{s=0}^{\infty} \alpha_{s}(r) x^{2 s}, \quad \mathbf{E}_{2 r+1}(x)=\frac{2}{(2 r+1) \pi} \sum_{s=0}^{\infty} \frac{2 r-2 s-1}{2 r-1} \alpha_{s}(r) x^{2 s}
$$


where $\alpha_{0}(r)=1$, and

$$
\alpha_{s}(r)=\frac{1}{\left(4 r^{2}-3^{2}\right)\left(4 r^{2}-5^{2}\right) \ldots .\left\{4 r^{2}-(2 s+1)^{2}\right\}} \quad(s>0) .
$$

Using the inequality

$$
\left|\frac{\alpha_{s}(r)}{\alpha_{s-1}(r)}\right| \leqslant \frac{1}{|4 r-1|}
$$

we deduce that if $x$ is fixed and $r \rightarrow \infty$, then

$$
\mathbf{E}_{2 r}(x) \sim \frac{2 x}{\left(4 r^{2}-1\right) \pi}, \quad \mathbf{E}_{2 r+1}(x) \sim \frac{2}{(2 r+1) \pi} .
$$

The corresponding homogeneous form of (2.01) has the Bessel functions $J_{r}(x)$ and $Y_{r}(x)$ as solutions. For fixed $x$ and large $r$, we have

$$
J_{r}(x) \sim \frac{1}{(2 \pi r)^{1 / 2}}\left(\frac{e x}{2 r}\right)^{r}, \quad Y_{r}(x) \sim-\left(\frac{2}{\pi r}\right)^{1 / 2}\left(\frac{2 r}{e x}\right)^{r}
$$

Thus ultimately $J_{r}(x)$ decays more rapidly than $\mathbf{E}_{r}(x)$, and $\left|Y_{r}(x)\right|$ grows rapidly. In consequence, both simple forward recurrence and simple backward recurrence are unstable methods for generating $\mathbf{E}_{r}(x)$ from (2.01) when $r>x$.

With

$$
f_{r}=J_{r}(x), \quad g_{r}=Y_{r}(x), \quad h_{r}=\mathbf{E}_{r}(x),
$$

the conditions of section 2 are satisfied, provided that $J_{0}(x) \neq 0$. Let us apply the method of section 4 to a specific example, say the computation of $\mathbf{E}_{r}(x)$ for $x=1, r=1(1) 10$, correct to within 2 units of the eighth decimal place. We suppose that we are given $\mathbf{E}_{0}(1)=-0.568656627$, this value having been extracted from [9] and confirmed by evaluation of the first of (6.01).

Beginning with $p_{0}=0, p_{1}=1$, and $e_{0}=\mathbf{E}_{0}(1)$, values of $p_{r}$ and $e_{r}$ were generated by use of (4.05). They are recorded in the upper part of table 1, correct to 9 significant figures. After passing the last of the given values of $r$, namely 10 , the "test function" $p_{10} e_{r} /\left(p_{r} p_{r+1}\right)$ was computed. This falls below the value $2 \times 10^{-8}$ for the first time when $r=14$. In accordance with the criterion of section 4 this is the value ${ }^{4}$ to be assigned to $N$. The column of values $y_{r}^{(14)}$ was then generated by backward use of (4.04), beginning with $y_{14}^{(14)}=0$. For $r=1(1) 10$ these are the wanted approximations to $\mathbf{E}_{r}(1)$.

To test the accuracy of the results, the computations were repeated for $N=32$ and $N=34$, using a time-sharing automatic computer and working to 36 floating binary figures, with an exponent of 12 binary figures. As further checks the values for $r=1(1) 5$ were compared with the 10-decimal, values given in [9], and the values for $r=10$ and 11 computed from the expansions (6.01). The full results of these computations are not included here, but the digits in $y_{r}^{(14)}$ which differ from those in the more accurate values of $\mathbf{E}_{r}(1)$ are printed in italic type, and the difference $\epsilon_{r}^{(14)}$ between the two values is recorded, in units of the 9 th decimal place, in the penultimate column of the upper part of table 1 . As expected, this error does not exceed $2 \times 10^{-8}$ in absolute value within the wanted range $r=1(1) 10$.

${ }^{4}$ The more precise criterion of section 5 requires that the sum of $p_{10} e_{r} /\left(p_{r} p_{r+1}\right)$ and all subsequent values in this column be less than $2 \times 10^{-8}$. Inspection of table 1 indicates that this also is satisfied for $N=14$. This would not be the case, however, if we reduced our error tolerance in $y_{r}$ from $2 \times 10^{-8}$ to $1 \times 10^{-8}$. 
TABLE 1. Anger-Weber function $\mathbf{E}_{r}(1)$

\begin{tabular}{|c|c|c|c|c|c|c|}
\hline$r$ & $p_{r}$ & $e_{r}$ & $\frac{p_{10} e_{r}}{p_{r} p_{r+1}}$ & $y_{r}^{(14)}$ & $10^{9} \epsilon_{r}^{(14)}$ & $10^{9} E_{14} p_{r}$ \\
\hline $\begin{array}{r}0 \\
1 \\
2 \\
3 \\
4 \\
5 \\
6 \\
7 \\
8 \\
9 \\
10 \\
11 \\
12 \\
13 \\
14 \\
15 \\
16\end{array}$ & $\begin{array}{rr} & 0 \\
& 1 \\
& 2 \\
& 7 \\
& 40 \\
& 313 \\
& 3090 \\
36767 \\
511648 \\
8149601 \\
1461 \quad 81170 \\
2.91547 \quad 380 \times 10^{9} \\
6.39942 \quad 424 \times 10^{10} \\
1.53294 \quad 634 \times 10^{12} \\
3.97926 \quad 106 \times 10^{13} \\
1.11266 \quad 015 \times 10^{15} \\
3.33400 \quad 119 \times 10^{16}\end{array}$ & $\begin{array}{rl}-0.56865 & 6627 \\
0.70458 & 2918 \\
0.70458 & 2918 \\
9.61725 & 973 \\
9.61725 & 973 \\
4.08141 & 237 \times 10^{2} \\
4.08141 & 237 \times 10^{2} \\
4.72213 & 396 \times 10^{4} \\
4.72213 & 396 \times 10^{4} \\
1.04236 & 156 \times 10^{7} \\
1.04236 & 156 \times 10^{7} \\
3.72252 & 015 \times 10^{9} \\
3.72252 & 015 \times 10^{9} \\
1.95553 & 042 \times 10^{12} \\
1.95553 & 042 \times 10^{12} \\
1.41863 & 843 \times 10^{15}\end{array}$ & $\begin{array}{l}3.6 \times 10^{-3} \\
2.9 \times 10^{-3} \\
5.5 \times 10^{-6} \\
4.7 \times 10^{-6} \\
6.5 \times 10^{-9} \\
5.6 \times 10^{-9}\end{array}$ & $\begin{array}{rl}0.43816 & 2436 \\
.17174 & 1955 \\
.24880 & 5382 \\
.04785 & 0795 \\
.13400 & 0978 \\
.01891 & 9443 \\
.09303 & 2343 \\
.01029 & 3811 \\
.07166 & 8637 \\
.00650 & 2117 \\
.05837 & 3706 \\
.00447 & 9865 \\
.04914 & 3054 \\
.00000 & 0000\end{array}$ & $\begin{array}{r}1 \\
12 \\
240 \\
5279 \\
126445\end{array}$ & $\begin{array}{r}1 \\
12 \\
240 \\
5279 \\
126444\end{array}$ \\
\hline$r$ & $p_{r}$ & $e_{r}$ & $\frac{e_{r}}{p_{r} p_{r+1}}$ & & & \\
\hline $\begin{array}{l}14 \\
15 \\
16 \\
17 \\
18 \\
19\end{array}$ & $\begin{array}{ll}3.97926 & 106 \times 10^{13} \\
1.11266 & 015 \times 10^{15} \\
3.33400 & 119 \times 10^{16} \\
1.06576 & 772 \times 10^{18} \\
3.62027 & 625 \times 10^{19} \\
1.30223 & 368 \times 10^{21}\end{array}$ & $\begin{array}{ll}1.95553 & 042 \times 10^{12} \\
1.41863 & 843 \times 10^{15} \\
1.41863 & 843 \times 10^{15} \\
1.35839 & 625 \times 10^{18} \\
1.35839 & 625 \times 10^{18}\end{array}$ & $\begin{array}{r}4.41672 \times 10^{-17} \\
3.82422 \times 10^{-17} \\
0.00399 \times 10^{-17} \\
.00352 \times 10^{-17} \\
.00000 \times 10^{-17}\end{array}$ & \multicolumn{3}{|c|}{$E_{14}=8.24845 \times 10^{-17}$} \\
\hline
\end{tabular}

It is of interest to apply the expansions of section 5 to this example. The necessary computations for evaluating the expansion (5.02) are given in the lower part of table 1, and the values of $10^{9} E_{14} p_{r}$ appear in the final column of the upper part of the same table. They agree with $10^{9} \epsilon_{r}^{(14)}$ to within a unit.

EXAMPle 2. Struve functions.

The function $\mathbf{H}_{r}(x)$ satisfies eq (2.01) with

$$
a_{r}=c_{r}=1, \quad b_{r}=\frac{2 r}{x}, \quad d_{r}=\frac{\left(\frac{1}{2} x\right)^{r}}{\sqrt{\pi} \Gamma\left(r+\frac{3}{2}\right)} .
$$

For fixed $x$ and large $r$ we have $([8]$, sec. 10.4)

$$
\mathbf{H}_{r}(x) \sim \frac{x}{\sqrt{2} \pi r}\left(\frac{e x}{2 r}\right)^{r}
$$

Since the complementary functions of the difference equation are again the Bessel functions $J_{r}(x)$ and $Y_{r}(x)$, the conditions of section 2 are satisfied, provided that $J_{0}(x) \neq 0$.

Let us evaluate $\mathbf{H}_{r}(0.1)$ to 8 significant figures for all positive integer values of $r$ such that $\left|\mathbf{H}_{r}(0.1)\right|$ exceeds $1 / 2 \times 10^{-30}$.

The computations are shown in table 2 . The value

$$
e_{0}=\mathbf{H}_{0}(0.1)=0.0635912700
$$


TABLE 2. Struve function $\mathbf{H}_{r}(0.1)$

\begin{tabular}{|c|c|c|c|c|c|c|c|}
\hline$r$ & $p_{r}$ & & $d_{r}$ & $e_{r}$ & $\frac{e_{r}}{p_{r} p_{r+1}}$ & $y_{r}^{(15)}$ & $\frac{10^{9} \epsilon_{r}^{(15)}}{y_{r}^{(15)}}$ \\
\hline 0 & 0 & 0.63661 & 9772 & $0.06359 \quad 12700$ & & & \\
\hline 1 & 1 & 2.12206 & $591 \times 10^{-2}$ & .0423706109 & & $2.12065160 \times 10^{-3}$ & 0 \\
\hline 2 & 20 & 4.24413 & $182 \times 10^{-4}$ & .0338823473 & & $4.24211 \quad 125 \times 10^{-5}$ & 0 \\
\hline 3 & 799 & 6.06304 & $546 \times 10^{-6}$ & .0290379740 & & $6.06080029 \times 10^{-7}$ & -1 \\
\hline 4 & 47920 & 6.73671 & $718 \times 10^{-8}$ & $.02580 \quad 97391$ & & $6.73467605 \times 10^{-9}$ & 0 \\
\hline 5 & 3832801 & 6.12428 & $835 \times 10^{-10}$ & .0234624212 & & $6.12271820 \times 10^{-11}$ & 2 \\
\hline 6 & 383232180 & 4.71099 & $104 \times 10^{-12}$ & .0216570178 & & $4.70994424 \times 10^{-13}$ & 4 \\
\hline 7 & $4.59840288 \times 10^{10}$ & 3.14066 & $069 \times 10^{-14}$ & .0202128155 & & $3.14004492 \times 10^{-15}$ & 4 \\
\hline 8 & $6.43738080 \times 10^{12}$ & 1.84744 & $746 \times 10^{-16}$ & .0190235432 & & $1.84712338 \times 10^{-17}$ & -1 \\
\hline 9 & $1.02993494 \times 10^{15}$ & 9.72340 & $768 \times 10^{-19}$ & .0180220955 & & $9.72186442 \times 10^{-20}$ & 0 \\
\hline 10 & $1.85381852 \times 10^{17}$ & 4.63019 & $413 \times 10^{-21}$ & .0171637415 & & $4.62952313 \times 10^{-22}$ & 4 \\
\hline 11 & $3.70753405 \times 10^{19}$ & 2.01312 & $788 \times 10^{-23}$ & .0164173675 & & $2.01285948 \times 10^{-24}$ & 4 \\
\hline 12 & $8.15638953 \times 10^{21}$ & 8.05251 & $152 \times 10^{-26}$ & .0157605733 & & $8.05151746 \times 10^{-27}$ & 2 \\
\hline 13 & $1.95749641 \times 10^{24}$ & 2.98241 & $167 \times 10^{-28}$ & .0151767673 & $1.5 \times 10^{-53}$ & $2.98206890 \times 10^{-29}$ & -1 \\
\hline 14 & $5.08940910 \times 10^{26}$ & 1.02841 & $782 \times 10^{-30}$ & .0146533634 & $2.0 \times 10^{-58}$ & $1.02829540 \times 10^{-31}$ & 11520 \\
\hline 15 & $1.42501497 \times 10^{29}$ & 3.31747 & $684 \times 10^{-33}$ & .0141806180 & $2.3 \times 10^{-63}$ & 0 & \\
\hline 16 & $4.27499402 \times 10^{31}$ & & & & & & \\
\hline
\end{tabular}

was extracted from [9], and confirmed by evaluation of the expansion

$$
\mathbf{H}_{r}(x)=\left(\frac{1}{2} x\right)^{r+1} \sum_{s=0}^{\infty} \frac{(-)^{s}\left(\frac{1}{2} x\right)^{2 s}}{\Gamma\left(s+\frac{3}{2}\right) \Gamma\left(s+r+\frac{3}{2}\right)} .
$$

The largest of the wanted values of $r$ was determined by the criterion

$$
\left|e_{r} / p_{r+1}\right|>\frac{1}{2} \times 10^{-30} \quad \text { and } \quad\left|e_{r+1} / p_{r+2}\right| \leqslant \frac{1}{2} \times 10^{-30}
$$

compare (5.03). This gave $r=13$. Next, we have from (5.01), (5.02), and (5.03),

$$
\frac{\epsilon_{r}^{(N)}}{y_{r}}=\frac{p_{r} p_{r+1}}{e_{r}} \frac{e_{N}}{p_{N} p_{N+1}}
$$

From table 2 we see that the right of this relation is an increasing function of $r$, hence $N$ is the least value for which

$$
\frac{e_{N}}{p_{N} p_{N+1}} \leqslant\left(\frac{1}{2} \times 10^{-8}\right) \frac{e_{13}}{p_{13} p_{14}} .
$$

From the entries in the column headed $e_{r} /\left(p_{r} p_{r+1}\right)$ we see immediately that this gives $N=15$.

The values of $y_{r}^{(15)}$, computed from (4.04), appear in the penultimate column of the table. For $r \leqslant 13$ they are the required approximations to $\mathbf{H}_{r}(0.1)$. Again, more accurate values were obtained by automatic computation with a higher value of $N(26)$, and also by evaluation. of the expansion (6.06) for $r=1(1) 15$. In the final column the relative error $\epsilon_{r}^{(15)} / y_{r}^{(15)}$ is given in units of the 9 th decimal place. As expected, it lies within the stipulated limit $1 / 2 \times 10^{-8}$ in the required range.

\section{Propagation of Rounding Errors}

In addition to the truncation error $\epsilon_{r}^{(N)}$ which has been analyzed in sections 4 and 5 , the other possible sources of error in the final solution are the rounding errors introduced during the calculations. Since the computing process is essentially the solution of a finite system of linear algebraic equations, the nature of the transmission of these errors is available from general theory [10] 
chapter $4 ;$ [6], chapter 9. However, because of special features of the present problem, including the fact that in our form of elimination the absolute values of the multipliers are not bounded by unity, some comments on the effects of rounding errors may be helpful.

Consider first the computation of the sequence $p_{r}$. From the conditions $p_{0}=0, p_{1}=1$, we see that in terms of the fundamental solutions $f_{r}$ and $g_{r}$ of section 2

$$
p_{r}=\left(f_{0} g_{r}-g_{0} f_{r}\right) /\left(f_{0} g_{1}-g_{0} f_{1}\right)
$$

the denominator here necessarily being nonzero since $f_{r}$ and $g_{r}$ are independent solutions of the difference equation. By hypothesis, $f_{0} \neq 0$; therefore $p_{r}$ always contains a multiple of $g_{r}$. And since $f_{r} / g_{r} \rightarrow 0$ as $r \rightarrow \infty, p_{r}$ ultimately becomes proportional to $g_{r}$ when a fixed number of significant figures is maintained in the computations.

Each rounding error in the formation of the $p_{r}$ can be regarded as introducing unwanted small multiples of $f_{r}$ and $g_{r}$. Ultimately, the former dies out in comparison with the latter; the error is then propagated at the same rate as $p_{r}$ itself. Before this stage is attained, however, some loss of accuracy is possible. If the value of $\left|f_{0}\right|$ is unduly small compared with $\left|g_{0} f_{1} / g_{1}\right|$, then from (7.01) we see that initially $p_{r}$ behaves like a multiple of $f_{r}$. But the rounding errors are still propagated in proportion to $g_{r}$, and this generally causes a steady loss of significant figures. The loss ceases when the term $f_{0} g_{r}$ in (7.01) overtakes $g_{0} f_{r}$ in magnitude, at which stage the computation becomes completely stable.

It should be realized that this loss of accuracy is not attributable to the method of computation, but to the fact that, as a rule, the whole problem is ill-posed when $\left|f_{0}\right|$ is small compared with $\left|g_{0} f_{r} / g_{r}\right|$ for at least one value of $r$. For from (2.06) we see that

$$
\left|\frac{\delta y_{r}}{y_{r}}\right|=\left|\frac{f_{r} \delta k}{f_{0} y_{r}}\right| \gg\left|\frac{g_{r} \delta k}{g_{0} y_{r}}\right|
$$

where $\delta y_{r}$ is the change in $y_{r}$ consequent upon an arbitrary change $\delta k$ in the value of $k$. Since $\left|g_{r} / g_{0}\right|$ is generally large compared with $\left|y_{r}\right|$ (see (2.04)), the relative error in $y_{r}$ is very sensitive to rounding errors in the given value of $k$.

Examples 1 and 2 of section 6 would be ill-posed in this way if the chosen value of $x$ were close to a zero of $J_{0}(x)$, say $x=5.52$. This would become apparent at the beginning of the computations: the early $p_{r}$ would diminish in size, in contrast to the behavior they exhibit in tables 1 and 2.

The difficulty could be overcome in these and other examples by carrying out the computation of $k$ and $p_{r}$ to higher precision, and making the necessary prolongation of the recurrences until the criteria of sections 4 and 5 for terminating them are met.

If the value of $y_{1}$ can be found, however, a preferable alternative is to apply the algorithm of sections 3 and 4 with the given $y_{1}$ as normalizing value, instead of $y_{0}=k$. In effect, this means that the recurrences (4.05) are begun with $p_{1}=0, p_{2}=1$, and $e_{1}=y_{1}$. Subsequently the value of $y_{0}$ can be computed from $y_{1}$ and $y_{2}$ by a single backward application of (2.01).

The other part of the elimination process is the computation of the right-hand sides $e_{r}$. From (4.05) we see that in the inhomogeneous case instability could arise from this source if there were persistent heavy cancellation between $a_{r} e_{r-1}$ and $d_{r} p_{r}$. No naturally occurring examples of this phenomenon have been encountered so far, however.

Lastly, we see from (4.04) that a rounding error introduced in $y_{s}^{(N)}$ during the back-substitution is multiplied by the factor $p_{r} / p_{s}$ when it is transmitted to $y_{r}^{(N)}(r<s)$. Except when the problem is ill-posed, this factor decays with diminishing $r$ at a faster rate than $y_{r}^{(N)}$ itself, because $p_{r}$ contains a substantial multiple of $g_{r}$, and $y_{r}$ contains no multiple of this function.

Summarizing this section, we have shown that unstable transmission of rounding errors can occur only when the original problem is ill-posed or when heavy cancellation takes place during the calculation of $e_{r}$ from the second of (4.05). 


\section{Comparison With the Algorithms of Miller and Shintani}

In section 4 we solved the set of eqs (3.01) and (3.02) by eliminating the variables in the order $y_{1}^{(N)}, y_{2}^{(N)}, \ldots ., y_{N-2}^{(N)}$ : we may call this forward elimination. Suppose now that these variables are eliminated in the reverse order: backward elimination. The resulting set of pivotal equations can be expressed in the form

$$
u_{r+1}^{(N)} y_{r}^{(N)}-u_{r}^{(N)} y_{r+1}^{(N)}=v_{r}^{(N)} \quad(r=N-2, N-3, \ldots, 0)
$$

where the quantities $u_{r}^{(N)}$ and $v_{r}^{(N)}$ are defined by

$$
u_{N-1}^{(N)}=1, \quad u_{N-2}^{(N)}=b_{N-1} / a_{N-1}, \quad v_{N-2}^{(N)}=d_{N-1} / a_{N-1},
$$

and

$$
u_{r-1}^{(N)}=\frac{b_{r} u_{r}^{(N)}-c_{r} u_{r+1}^{(N)}}{a_{r}}, \quad v_{r-1}^{(N)}=\frac{c_{r} v_{r}^{(N)}+d_{r} u_{r}^{(N)}}{a_{r}}, \quad(r<N-1)
$$

(It should be observed that $u_{r}^{(N)}$ and $v_{r}^{(N)}$ depend on $N$ as well as $r$, unlike the $p_{r}$ and $e_{r}$ of section 4.)

The last of eqs (8.01) is used to begin the back-substitution. It yields

$$
y_{1}^{(N)}=\left(u_{1}^{(N)} k-v_{0}^{(N)}\right) / u_{0}^{(N)},
$$

where $k$ is again the given value of $y_{0}$. Then $y_{2}^{(N)}, y_{3}^{(N)}, \ldots, y_{N-1}^{(N)}$ may be computed by successive application of (8.01) with $r=1,2, \ldots ., N-2$.

Thus the elimination process consists of constructing a sequence $u_{r}^{(N)}$ which satisfies the homogeneous form of the given difference equation $(2.01)$ and the conditions $u_{N}^{(N)}=0, u_{N-1}^{(N)}=1$. This is exactly the first stage of Miller's algorithm [1], [3]: the $u_{r}^{(N)}$ are the so-called trial values. And in the homogeneous case, given by $d_{r}=0$, all the quantities $v_{r}^{(N)}$ vanish, causing the formulas (8.01) for back-substitution to reduce to

$$
y_{r}^{(N)}=\frac{u_{r}^{(N)}}{u_{r-1}^{(N)}} y_{r-1}^{(N)}=\frac{u_{r}^{(N)}}{u_{r-1}^{(N)}} \frac{u_{r-1}^{(N)}}{u_{r-2}^{(N)}} \ldots \frac{u_{1}^{(N)}}{u_{0}^{(N)}} y_{0}^{(N)}=\frac{k}{u_{0}^{(N)}} u_{r}^{(N)} .
$$

This is the second stage of the Miller algorithm: $k / u_{0}^{(N)}$ is the normalizing factor.

Accordingly, in the homogeneous case the Miller recurrence algorithm can be regarded as the solution of the set of equations (3.01) and (3.02), with $\mathrm{d}_{\mathrm{r}}=0$, by backward elimination. In the inhomogeneous case the solution by backward elimination, described above, can be regarded as a generalization of the Miller algorithm.

Compared with the forward elimination process of section 4, the Miller algorithm suffers from the disadvantages that it does not determine automatically the correct value of $N$, and if a second value of $N$ is used as a check on the adequacy of the original value, then the computations must begin afresh. The advantage of the Miller algorithm is that the process of back-substitution is less laborious; this advantage is restricted to the homogeneous case, however, and is offset if more than one trial value of $N$ has to be used.

The method Shintani [5] ${ }^{5}$ has developed for solving second-order linear difference equations in the homogeneous case consists of the use of the Miller algorithm preceded by two forward recurrence processes to determine the optimum value of $N$. In the present notation, Shintani takes $a_{r}=1$ and $d_{r}=0$. His formulas for forward recurrence are given by ([5], Theorem 1) 


$$
P_{r+1}(\nu)=b_{r+1} P_{r}(\nu)-c_{r} P_{r-1}(\nu)
$$

where $\nu=0$ or 1 , and

$$
P_{-1}(0)=0, \quad P_{0}(0)=1 ; \quad P_{0}(1)=0, \quad P_{1}(1)=1 .
$$

It is easily verified, for example, that the quantities $P_{r}(0)$ appear when the forward elimination procedure is applied to eqs (4.01) with $d_{r}=0$ and the multipliers chosen in such a way that the constant value $-k$ is preserved on the right-hand sides. The resulting pivotal equations are in fact

$$
-P_{r}(0) y_{r}^{(N)}+c_{r} P_{r-1}(0) y_{r+1}^{(N)}=-k . \quad(r=1,2, \ldots, N-1) .
$$

In our notation

$$
P_{r}(0)=c_{1} c_{2} \ldots c_{r} p_{r+1} .
$$

From the computational standpoint, the evaluation of Shintani's sequence $P_{r}(0)$ may be compared with the evaluation of our sequence $p_{r}$, the evaluation of his $P_{r}(1)$ with our $e_{r}$, and the application of the Miller algorithm with our process of back-substitution. In the first stage the computing effort is identical, but in the second and third stages our method requires considerably less effort.

\section{More General Form of Normalizing Condition}

Let us consider now the solution of the difference eq (2.01) when (2.05) is replaced by the more general normalizing condition

$$
m_{0} y_{0}+m_{1} y_{1}+m_{2} y_{2}+\ldots=k,
$$

in which $m_{0}, m_{1}, \ldots$, and $k$ are given constants. We again suppose that the general solution of (2.01) has the form (2.02), but instead of the conditions imposed on $f_{r}, g_{r}$, and $h_{r}$ in section 2, we assume that

$$
\left|\sum_{r=0}^{N} m_{r} g_{r}\right| \rightarrow \infty \text { as } N \rightarrow \infty
$$

and

$$
\sum_{r=0}^{\infty} m_{r} f_{r}=F, \quad \sum_{r=0}^{\infty} m_{r} h_{r}=H
$$

where $F$ and $H$ are finite, and $F \neq 0$. Then (2.01) has a unique solution fulfilling (9.01). It is given by

$$
y_{r}=\frac{k-H}{F} f_{r}+h_{r}
$$

compare (2.06).

The obvious extension of the approach of section 3 is to solve the system of linear algebraic equations given by

and

$$
\begin{aligned}
a_{r} y_{r-1}^{(N)}-b_{r} y_{r}^{(N)}+c_{r} y_{r+1}^{(N)} & =d_{r} \quad(r=1,2, \ldots, N-1), \\
\sum_{r=0}^{N} m_{r} y_{r}^{(N)} & =k,
\end{aligned}
$$

$$
y_{N}^{(N)}=0
$$


THEOREM 2. In addition to the other conditions of this section, assume that for all sufficiently large $\mathrm{N}$ the system of equations (9.05), (9.06), and (9.07) has a solution, that $\mathrm{g}_{\mathrm{N}} \neq 0$, and that

$$
\frac{f_{N}}{g_{N}} \sum_{r=0}^{N} m_{r} g_{r} \rightarrow 0, \quad \frac{h_{N}}{g_{N}} \sum_{r=0}^{N} m_{r} g_{r} \rightarrow 0, \quad(N \rightarrow \infty) .
$$

Then if $\mathrm{r}$ is fixed and $\mathrm{N} \rightarrow \infty, \mathrm{y}_{\mathrm{r}}^{(\mathrm{N})} \rightarrow \mathrm{y}_{\mathrm{r}}$.

This result may be established by expressing $y_{r}^{(N)}$ in the form

$$
y_{r}^{(N)}=A_{N} f_{r}+B_{N} g_{r}+h_{r} .
$$

Using (9.06) and (9.07), we find that

$$
A_{N}=\frac{h_{N} \sum_{r=0}^{N} m_{r} g_{r}-g_{N}\left(\sum_{r=0}^{N} m_{r} h_{r}-k\right)}{g_{N} \sum_{r=0}^{N} m_{r} f_{r}-f_{N} \sum_{r=0}^{N} m_{r} g_{r}}, \quad B_{N}=\frac{f_{N}\left(\sum_{r=0}^{N} m_{r} h_{r}-k\right)-h_{N} \sum_{r=0}^{N} m_{r} f_{r}}{g_{N} \sum_{r=0}^{N} m_{r} f_{r}-f_{N} \sum_{r=0}^{N} m_{r} g_{r}} .
$$

In consequence of the assumed conditions, the denominators are asymptotic to $F g_{N}$ as $N \rightarrow \infty$. Hence $A_{N} \rightarrow(k-H) / F$. Next, the assumed conditions imply that $f_{N} / g_{N}$ and $h_{N} / g_{N}$ both tend to zero. Hence $B_{N} \rightarrow 0$. Comparison of (9.04) and (9.09) completes the proof.

When the forward elimination process of section 4 is applied to eqs (9.05), (9.06), and (9.07), the following pivotal equations are obtained:

$$
p_{r+1} y_{r}^{(N)}-p_{r} y_{r+1}^{(N)}+q_{r}\left(\sum_{s=r+1}^{N-1} m_{s} y_{s}^{(N)}\right)=e_{r} \quad(r=0,1, \ldots, N-1),
$$

(compare (4.04)), where

and, if $r \geqslant 1$

$$
\begin{aligned}
& q_{0}=1, \quad q_{r}=\frac{a_{1} a_{2} \ldots a_{r}}{c_{1} c_{2} \ldots c_{r}} \quad(r \geqslant 1), \\
& p_{0}=0, \quad p_{1}=m_{0}, \quad e_{0}=k,
\end{aligned}
$$

$$
p_{r+1}=\frac{b_{r} p_{r}-a_{r} p_{r-1}}{c_{r}}+q_{r} m_{r}, \quad e_{r}=\frac{a_{r} e_{r-1}-d_{r} p_{r}}{c_{r}}
$$

In consequence of (9.07), the final equation of the form (9.10) reduces to

$$
p_{N} y_{N-1}^{(N)}=e_{N-1}
$$

This yields the value of $y_{N-1}^{(N)}$; thence $y_{N-2}^{(N)}, y_{N-3}^{(N)}, \ldots, y_{0}^{(N)}$ may be computed from $(9.10)$ by backsubstitution.

The value of $N$ may be determined in a similar way to that suggested in section 4 . Suppose, for example, that all nonvanishing values of $y_{r}$ are needed to a fixed number of decimal places, 
$D$, say-a common form of requirement with the present type of normalizing condition. Then $N$ is determined by the condition

$$
\left|e_{N} / p_{N+1}\right|<\frac{1}{2} \times 10^{-D},
$$

provided that $\left|p_{r}\right| \leqslant\left|p_{N}\right|$ when $r \leqslant N$, and also

$$
\left|p_{r} / q_{r-1}\right|>\left|m_{r}\right|,\left|m_{r+1}\right|, \ldots .,\left|m_{N}\right| .
$$

Example 3. Bessel functions.

Let us evaluate $J_{0}(x), J_{1}(x), \ldots$, for $x=5$ to 5 decimal places, by use of the relations

$$
J_{r-1}(x)-(2 r / x) J_{r}(x)+J_{r+1}(x)=0,
$$

and

$$
J_{0}(x)+2 J_{2}(x)+2 J_{4}(x)+\ldots .=1 \text {. }
$$

In the present notation, we have

$$
\begin{gathered}
a_{r}=c_{r}=1, \quad b_{r}=2 r / x, \quad d_{r}=0, \\
m_{0}=1, \quad m_{1}=m_{3}=\ldots .=0, \quad m_{2}=m_{4}=\ldots=2, \quad k=1 .
\end{gathered}
$$

Accordingly, eqs (9.11) through (9.13) yield

and

$$
q_{r}=1, \quad e_{r}=1, \quad p_{0}=0, \quad p_{1}=1,
$$

$$
p_{r+1}=b_{r} p_{r}-p_{r-1}+m_{r} .
$$

Table 3 gives the values of $p_{r}$ correct to 6 significant figures. The criterion (9.15) suggests that $N$ be taken as the least value of $r$ for which $\left|p_{r+1}\right|>2 \times 10^{5}$. This gives ${ }^{6} N=14$. The column of values of $y_{r}^{(14)}$ is then generated upwards by use of $(9.10)$, starting with $y_{14}^{(14)}=0$. These are the required approximations to $J_{n}(5)$ : their differences, $\epsilon_{r}^{(14)}$, from the true values are recorded in the final column in units of the 5 th decimal place. The agreement is satisfactory.

TABLE 3. Bessel function $\mathrm{J}_{\mathbf{r}}(5)$

\begin{tabular}{r|r|r|c|r|r|r}
\hline \hline & & & & & & \\
& $b_{r}$ & $m_{r}$ & $p_{r}$ & $y_{r}^{(14)}$ & $\sum^{13} m_{s} y_{8}^{(14)}$ & $10^{5} \epsilon_{r}^{(14)}$ \\
\hline & & & & & & \\
0 & 0.0 & 1 & 0 & -0.17758 & & -2 \\
1 & 0.4 & 0 & 1 & -.32758 & 1.17758 & 0 \\
2 & 0.8 & 2 & 0.4 & .04655 & 1.17758 & 2 \\
3 & 1.2 & 0 & 1.32 & .36482 & 1.08448 & 1 \\
4 & 1.6 & 2 & 1.184 & .39123 & 1.08448 & 0 \\
5 & 2.0 & 0 & 2.5744 & .26114 & 0.30202 & 0 \\
6 & 2.4 & 2 & 3.9648 & .13105 & .30202 & 0 \\
7 & 2.8 & 0 & 8.94112 & .05338 & .03992 & 0 \\
8 & 3.2 & 2 & 21.0703 & .01841 & .03992 & 0 \\
9 & 3.6 & 0 & 60.4838 & .00552 & .00310 & 0 \\
10 & 4.0 & 2 & 196.671 & .00147 & .00310 & 0 \\
11 & 4.4 & 0 & 728.200 & .00035 & .00016 & 0 \\
12 & 4.8 & 2 & 2007.41 & .00008 & .00016 & 0 \\
13 & 5.2 & 0 & 13709.4 & .00001 & .00000 & 1 \\
14 & 5.6 & 2 & 68281.5 & .00000 & .00000 & 0 \\
15 & & & 368669. & & & \\
& & & & & & \\
\hline
\end{tabular}

${ }^{6}$ When the condition (9.16) is violated - as it is in this example near the beginning of the range-it would be safer in practice to take $N$ slightly higher than the value prẹdicted by $(9.15)$. 
It may be noted that estimates of the optimum value of $N$ for generating Bessel functions from । (9.17) and (9.18) by Miller's algorithm have been computed by Makinouchi [11] for $x=0.01(.01)$ $0.1(.1) 1(1) 10(10) 100$ and precisions of $9,10,18,20$, and 30 significant figures. These values were obtained by use of the asymptotic approximations (6.04) above. In constructing a program for generating the $J_{r}(x)$ for arbitrary $x$ and arbitrary precision, however, it would be simpler to determine the optimum $N$ by use of (9.15) (or (4.11)). The resulting gain would tend to offset the extra effort needed in applying the back-substitution relation (9.10) compared with the normalizing of the trial values in the Miller algorithm.

\section{Bounds for the Truncation Error}

In order to obtain strict bounds for the truncation error associated with the algorithm of section 9, we proceed as in section 5 . Write, temporarily,

$$
\eta_{r}=y_{r}^{(N+1)}-y_{r}^{(N)} .
$$

Then from (9.10) we obtain

$$
p_{r+1} \eta_{r}=p_{r} \eta_{r+1}-q_{r}\left(m_{r+1} \eta_{r+1}+\ldots+m_{N} \eta_{N}\right) \quad(r<N) .
$$

Therefore

$$
\left|\eta_{r}\right| \leqslant \rho_{r}\left(\left|\eta_{r+1}\right|+\left|\eta_{r+2}\right|+\ldots+\left|\eta_{N}\right|\right) \quad(r<N)
$$

where $\rho_{r}$ is the greater of

$$
\left|\frac{p_{r}-q_{r} m_{r+1}}{p_{r+1}}\right| \text { and }\left|\frac{q_{r}}{p_{r+1}}\right| \sup _{2 \leqslant s \leqslant \infty}\left|m_{r+s}\right| \text {. }
$$

Equations (9.07), (9.14), and (10.01) yield $\eta_{N}=e_{N} / p_{N+1}$. From this result and (10.03) we may verify that

$$
\left|\eta_{N-1}\right| \leqslant \rho_{N-1}\left|e_{N} / p_{N+1}\right|
$$

and thence by induction that

$$
\left|\eta_{r}\right| \leqslant \rho_{r}\left(1+\rho_{r+1}\right)\left(1+\rho_{r+2}\right) \ldots\left(1+\rho_{N-1}\right)\left|e_{N} / p_{N+1}\right| \quad(r \leqslant N-2) .
$$

The left-hand side of the last relation is $\left|y_{r}^{(N+1)}-y_{r}^{(N)}\right|$. Replacing $N$ by $N+1, N+2, \ldots$, in turn and summing, and applying Theorem 2 , we find that

$$
\left|\epsilon_{r}^{(N)}\right| \leqslant \rho_{r}\left(1+\rho_{r+1}\right)\left(1+\rho_{r+2}\right) \ldots\left(1+\rho_{N-1}\right) E_{N} \quad(r \leqslant N-2),
$$

where

$$
\epsilon_{r}^{(N)}=y_{r}-y_{r}^{(N)}
$$

and

$$
E_{N}=\left|\frac{e_{N}}{p_{N+1}}\right|+\left(1+\rho_{N}\right)\left|\frac{e_{N+1}}{p_{N+2}}\right|+\left(1+\rho_{N}\right)\left(1+\rho_{N+1}\right)\left|\frac{e_{N+2}}{p_{N+3}}\right|+\ldots,
$$

provided that the last series converges. Similarly

$$
\left|\epsilon_{N-1}^{(N)}\right| \leqslant \rho_{N-1} E_{N}, \quad\left|y_{N}\right| \leqslant E_{N} .
$$


The results (10.07) and (10.10) are strict bounds for the truncation error, in contrast to the expansion of section 5 which is exact (for the algorithm of sections 3 and 4). Often the bound (10.07) is a considerable overestimate. ${ }^{7}$ Thus in Example 3, the right-hand side of (10.07) or (10.10) has the following values for $N=14$, in units of the 5 th decimal place:

$568,237,29,12,3,1,1$, then zero for $r=7,8, \ldots ., 14$.

In consequence, if $N$ is determined by the criterion that for each required value of $r$ the right-hand sides of (10.07) and (10.10) must not exceed the specified tolerance in $y_{r}$, then the resulting value is perfectly safe but often unnecessarily high. Applied to Example 3, this criterion yields $N=18$, compared with the value 14 which we used and found to be quite adequate.

In the next section we give an alternative formulation of the algorithm of section 9. Although perhaps less elegant, it generally yields a sharper assessment of the truncation error than that of this section.

\section{Alternative Method for the General Normalizing Condition}

The algorithm of sections 3 and 4 can be applied to the problem of section 9 in the following way. First, we construct a solution $f_{r}$ of the homogeneous form of the given equation (2.01). The choice of this solution is arbitrary, provided that the first of (2.03) is satisfied. Then by means of an additional back-substitution we construct an arbitrary solution $h_{r}$ of (2.01) itself. The required solution $y_{r}$ may then be computed from (9.04), in which $k$ is defined by (9.01), and $F, H$ by (9.03). In the case when the given difference eq (2.01) is itself homogeneous, only the solution $f_{r}$ need be computed, and (9.04) reduces to

$$
y_{r}=(k / F) f_{r} .
$$

The simplest choice of the normalizing conditions needed for constructing $f_{r}$ and $h_{r}$ is given by

$$
f_{0}=1, \quad h_{0}=0 .
$$

The first of these may be an inconvenient or even impossible condition, however; in this event we may follow the suggestion given in section 7 and use instead

$$
f_{1}=1, \quad h_{1}=0 .
$$

To assess the truncation error in the final solution $y_{r}$, let $\varphi_{r}^{(N)}$ and $\theta_{r}^{(N)}$ be the truncation errors in the approximations $f_{r}^{(N)}$ and $h_{r}^{(N)}$ to $f_{r}$ and $h_{r}$; thus

$$
f_{r}=f_{r}^{(N)}+\varphi_{r}^{(N)}, \quad h_{r}=h_{r}^{(N)}+\theta_{r}^{(N)} .
$$

Bounds for $\varphi_{r}^{(N)}$ and $\theta_{r}^{(N)}$ are computable from the expansions of section 5. From (9.04) we have

$$
y_{r}=\frac{k-H_{N}-\tau_{N}}{F_{. N}+\sigma_{N}}\left(f_{r}^{(N)}+\varphi_{r}^{(N)}\right)+h_{r}^{(N)}+\theta_{r}^{(N)} \quad(r=0,1, \ldots, N),
$$

where $F_{N}, H_{N}$ are the computed quantities

$$
F_{N}=\sum_{r=0}^{N-1} m_{r} f_{r}^{(N)}, \quad H_{N}=\sum_{r=0}^{N-1} m_{r} h_{r}^{(N)}
$$

\footnotetext{
${ }^{7}$ This can be traced to the fact that over most of the range the second of the two quantities (10.04) is usually very much smaller than the first.
} 
and $\sigma_{N}, \tau_{N}$ assessable errors ${ }^{8}$

$$
\begin{aligned}
\sigma_{N} & =\sum_{r=0}^{N} m_{r} \varphi_{r}^{(N)}+\sum_{r=N+1}^{\infty} m_{r} f_{r}, \\
\tau_{N} & =\sum_{r=0}^{N} m_{r} \theta_{r}^{(N)}+\sum_{r=N+1}^{\infty} m_{r} h_{r} .
\end{aligned}
$$

If $F_{N} \neq 0$, then to the first order of small quantities the truncation error in the formula

$$
y_{r} \fallingdotseq \frac{k-H_{N}}{F_{N}} f_{r}^{(N)}+h_{r}^{(N)}
$$

is composed of three parts:

$$
\frac{k-H_{N}}{F_{N}} \varphi_{r}^{(N)}, \quad-\left\{\tau_{N}+\frac{\sigma_{N}\left(k-H_{N}\right)}{F_{N}}\right\} \frac{f_{r}^{(N)}}{F_{N}}, \quad \theta_{r}^{(N)}, \quad(r \leqslant N) .
$$

In the homogeneous case they reduce to two:

$$
\frac{k}{F_{N}} \varphi_{r}^{(N)}, \quad-\frac{k \sigma_{N}}{F_{N}^{2}} f_{r}^{(N)}, \quad(r \leqslant N)
$$

The first of (11.11) is the normalized multiple of the truncation error in the formula $f_{r} \fallingdotseq f_{r}^{(N)}$; the second of (11.11) is a fixed relative error arising from the approximate representation of the normalizing factor $k / F$ by $k / F_{N}$.

The equivalence of the method of this section to the algorithm of section 9 can be seen from the fact that the function on the right of (11.09) is exactly the solution of the set of eqs (9.05), (9.06), and (9.07).

EXAMPLE $4 .^{9}$

Let us compute to 5 decimal places the solution of the homogeneous equation

$$
(2 r-1) y_{r-1}-12 r y_{r}+(2 r+1) y_{r+1}=0,
$$

satisfying the condition

$$
\frac{1}{2} y_{0}+y_{1}+y_{2}+y_{3}+\ldots=1 \text {. }
$$

In the notation of sections 2 and 9 we have

$$
a_{r}=2 r-1, \quad b_{r}=12 r, \quad c_{r}=2 r+1, \quad d_{r}=0, \quad m_{0}=\frac{1}{2}, \quad m_{r}=1 \quad(r>0), \quad k=1 .
$$

The computations are shown in table 4 . Values of $p_{r}$ were generated from $p_{0}=0, p_{1}=1$, and (11.12) when $r>1$, correct to 6 significant figures. With $e_{0}=1$ (compare the first of (11.02)), we find from the second of (4.05) that $e_{r}=1 /(2 r+1)$. The least value of $r$ for which $e_{r} / p_{r+1}<\frac{1}{2} \times 10^{-5}$ is 7 ; in

${ }^{8}$ See also [7].

${ }^{9}[3]$, section 5 .

${ }^{10}$ An altemative way of estimating $N$ in this particular example is to observe that, except for small $r$, the wanted solution of (11.12) behaves roughly like $A \lambda^{-r}$, where $A$ is a constant and $\lambda^{2}-6 \lambda+1=0$, giving $\lambda \fallingdotseq 3+\sqrt{8}=5.8$. Assuming that $A$ is of order unity, as is reasonable in view of the condition (11.13), we find that $N \doteqdot 5 / \log _{10} \lambda \fallingdotseq 7$. This method of estimation is somewhat less certain than the one we have used, and it is not universally applicable. 
accordance with (4.12) this is the value ${ }^{10}$ to ascribe to $N$. The back-substitution process for the determination of $f_{r}^{(7)}$ is given by $f_{7}^{(7)}=0$, and

$$
p_{r+1} f_{r}^{(7)}=p_{r} f_{r+1}^{(7)}+e_{r} \quad(r=6,5, \ldots, 0)
$$

compare (4.04). Division of $f_{r}^{(7)}$ by $F_{7}=0.599069$, computed from the first of (11.06), yields the wanted approximations $y_{r}^{(7)}$ to $y_{r}$.

TABLE 4

\begin{tabular}{l|c|r|r|r}
\hline \hline$r$ & $p_{r}$ & \multicolumn{1}{c|}{$e_{r}$} & $f_{r}^{(7)}$ & \multicolumn{1}{c}{$y_{r}^{(7)}$} \\
\hline & & & & \\
0 & 0 & 1.000000 & 1.000000 & 1.66926 \\
1 & 1 & 0.333333 & 0.086107 & 0.14373 \\
2 & 4 & .200000 & .011094 & .01852 \\
3 & 18.6 & .142857 & .001587 & .00265 \\
4 & 92.8 & .111111 & .000238 & .00040 \\
5 & 480.467 & .090909 & .000037 & .00006 \\
6 & 2544.80 & .076923 & .000006 & .00001 \\
7 & 13687.7 & .066667 & .000000 & .00000 \\
8 & 74445.6 & & & \\
\hline
\end{tabular}

The example is now complete, but it is of interest to illustrate the error analysis of this section. Accordingly, the whole calculation was repeated twice, keeping four extra significant figures throughout. In the first repetition the same value $N=7$ was used. In the second repetition a new $N$ was determined by the condition $\left|e_{N} / p_{N+1}\right|<\frac{1}{2} \times 10^{-9}$; this gave $N=12$.

The results appear in table 5 . The column headed $10^{9} \epsilon_{r}^{(7)}$ gives the difference of $10^{9} y_{r}^{(7)}$ from the more accurate values $10^{9} y_{r}^{(12)}$. The next columns give $10^{9} \varphi_{r}^{(7)} / F_{7}$ and $-10^{9} \sigma_{7} f_{r}^{(7)} / F_{7}^{2}$; the value of $\varphi_{r}^{(7)}$ was obtained by subtracting $f_{r}^{(7)}$ from $f_{r}^{(12)}$, and $\sigma_{7}$ computed from (11.07), using the values of $f_{r}^{(12)}$ for $f_{r}$ when $r \geqslant 8$. As expected, the values of $10^{9} \epsilon_{r}^{(7)}$ are in good agreement with the sum of the entries on the same row in the following two columns.

TABLE 5

\begin{tabular}{|c|c|c|c|c|c|c|c|}
\hline$r$ & $f_{r}^{(12)}$ & $y_{r}^{(12)}$ & $f_{r}^{(7)}$ & $y_{r}^{(7)}$ & $10^{9} \epsilon_{r}^{(7)}$ & $10^{9} \frac{\phi_{r}^{(7)}}{\bar{F}_{7}}$ & $-10^{9} \frac{\sigma_{7} f_{r}^{(7)}}{F_{7}^{2}}$ \\
\hline 0 & 1.0000000000 & 1.669253684 & 1.0000000000 & 1.669257339 & -3655 & 0 & -3655 \\
\hline 1 & 0.0861068379 & 0.143734156 & $0.08610 \quad 68378$ & 0.143734471 & -315 & 0 & -315 \\
\hline 2 & .0110940183 & .018518731 & .0110940180 & .018518771 & -40 & 1 & -41 \\
\hline 3 & .0015871852 & .002649415 & .0015871839 & .002649418 & -3 & 2 & -6 \\
\hline 4 & .0002383677 & .000397896 & .0002383614 & .000397887 & 9 & 11 & -1 \\
\hline 5 & .0000368169 & $.00006 \quad 1457$ & .0000367845 & $.00006 \quad 1403$ & 54 & 54 & 0 \\
\hline 6 & .0000057914 & .000009667 & .0000056199 & .000009381 & 286 & 286 & 0 \\
\hline 7 & .0000009228 & .000001540 & .0000000000 & .000000000 & 1540 & 1540 & 0 \\
\hline 8 & .0000001485 & $.00000 \quad 0248$ & & & & & \\
\hline 9 & $.00000 \quad 00241$ & .000000040 & \multirow{4}{*}{\multicolumn{5}{|c|}{$\begin{aligned} F_{7} & =0.5990688055 \\
F_{12} & =0.5990701173\end{aligned}$}} \\
\hline 10 & .0000000039 & $.00000 \quad 0007$ & & & & & \\
\hline 11 & .0000000006 & .000000001 & & & & & \\
\hline 12 & .0000000000 & .000000000 & & & & & \\
\hline
\end{tabular}

\section{Summary}

In this paper we have described a new algorithm for computing the solution $y_{r}$ of any secondorder linear difference equation, homogeneous or inhomogeneous, which is applicable when simple forward recurrence (and possibly also backward recurrence) cannot be used because of instability. 
In the first part (secs. 2-8) we considered the case in which the wanted solution $y_{r}$ has a specified value at the beginning of the range $r=0$, and an appropriate convergence condition as $r \rightarrow \infty$. In this case the algorithm is based on the solution of a finite number, $N$, of simultaneous linear algebraic equations of tridiagonal form by forward elimination. As $N \rightarrow \infty$ the solution $y_{r}^{(N)}$ of these equations converges to $y_{r}$ (sec. 3). In sections 4 and 5 it was shown that during the process of computing $y_{r}^{(N)}$ the minimum value of $N$ necessary to achieve specified tolerance in $\left|y_{r}-y_{r}^{(N)}\right|$ emerges automatically. Analyses of the truncation error and of the propagation of rounding errors were made in sections 5 and 7 . The former leads to a convergent series expansion for $y_{r}$; the latter shows that the method of computation is quite stable, unless the problem itself is ill-posed. Numerical examples (sec. 6) illustrated the algorithm and confirmed the error analyses.

In section 8 it was shown that the well-known algorithm of J. C. P. Miller for the homogeneous case can be regarded as the computation of $y_{r}^{(N)}$ by backward elimination, taking a guessed value of $N$. It was also shown that the recent extension of Miller's algorithm by Shintani is related to the process of forward elimination.

In the second part of the paper (secs. 9-11) a more general form of normalizing condition for $y_{r}$ was considered. An extended form of the algorithm was developed in section 9 and applied to a numerical example in the same section. In section 10 bounds for the truncation error were given and discussed. In the concluding section (sec. 11) it was shown that the more general problem can also be solved by application of the original algorithm of sections 3 and 4 .

It is hoped that the results of this paper will prove to be of considerable usefulness in the computation of special functions from recurrence relations, in the solution of ordinary differential equations in Chebyshev series by Clenshaw's method, and in the solution of the discretized form of boundary-value problems in ordinary differential equations when one boundary is at infinity. In the last two connections, it may be possible to extend the present approach to difference equations of order higher than the second.

The writer thanks C. W. Clenshaw, G. F. Miller, and D. L. Yarmush for valuable comments on this work, and also his wife, Mrs. G. E. Olver, for carrying out the desk and automatic computations of the numerical examples, only a few of which are included in the paper. The automatic computation was supported by National Institutes of Health Grant No. NB05613.01.

\section{References}

[1] British Association for the Advancement of Science, Bessel functions - Part II, Mathematical Tables, v. 10. (Cambridge University Press, 1952.)

[2] Clenshaw, C. W., The numerical solution of linear differential equations in Chebyshev series, Proc. Camb. Philos. Soc. 53, 134-149 (1957).

[3] Olver, F. W. J., Error analysis of Miller's recurrence algorithm, Math. Comp. 18, 65-74 (1964).

[4] Gautschi, W., Computational aspects of three-term recurrence relations, S.I.A.M. Rev. 9, 24-82 (1967).

[5] Shintani, H., Note on Miller's recurrence algorithm, J. Sci. Hiroshima Univ. Ser. A-I 29, 121-133 (1965).

[6] Fox, L., The numerical solution of two-point boundary problems in ordinary differential equations. (Oxford University Press, 1957.)

[7] Olver, F. W. J., Bounds for the solutions of second-order linear difference equations, J. Res. NBS.

[8] Watson, G. N., Theory of Bessel functions, Second edition. (Cambridge University Press, 1944.)

[9] Robinson, C., A numerical and analytical investigation of Struve's function. Thesis, London Univ. (1948).

[10] Wilkinson, J. H., The algebraic eigenvalue problem. (Oxford University Press, 1965.)

[11] Makinouchi, S., Note on the recurrence techniques for the calculation of Bessel functions $J_{v}(x)$, Tech. rep. Osaka Univ. 15, 185-201 (1965).

[12] Oliver, J., Relative error propagation in the recursive solution of linear recurrence relations, Num. Math. 9, 323-340 (1967).

(Paper 71B2\&3-206) 